\title{
IoT-based electricity energy monitoring system at Universiti Teknikal Malaysia Melaka
}

\author{
Meysam Shamshiri, Chin Kim Gan, Kyairul Azmi Baharin, Mohd Arif Mohd Azman \\ Energy and Power Systems Research Group-CeRIA, Faculty of Electrical Engineering, Universiti Teknikal Malaysia \\ Melaka, 76100 Durian Tunggal, Melaka, Malaysia
}

\begin{abstract}
Article Info
Article history:

Received Jul 24, 2018

Revised Jan 7, 2019

Accepted Apr 5, 2019

Keywords:

Energy efficiency

Energy monitoring

Internet of Things

ABSTRACT

Electricity bill is one of the major operating expenses in most of the commercial buildings and industrial plants. Thus, the buildings' energy management system is an essential element that should be utilized to optimize the energy usage and hence, contributes to carbon footprint reduction. To achieve this, one needs to first understand how the energy is being used in the buildings before any saving measures can be identified and proposed. Therefore, this paper presents the development of an Internet of Things (IoT) enabled device that can communicate with different digital energy meters through modbus protocol. The prototype has been successfully installed in three locations in the main campus of Universiti Teknikal Malaysia Melaka (UTeM). The proposed solution enables the campus-wide energy usage to be monitored and stored efficiently and economically as opposed to the capital-intensive SCADA system.
\end{abstract}

Copyright @ 2019 Institute of Advanced Engineering and Science. All rights reserved.

\section{Corresponding Author:}

Chin Kim Gan,

Energy and Power Systems Research Group-CeRIA,

Faculty of Electrical Engineering, Universiti Teknikal Malaysia Melaka,

76100 Durian Tunggal, Melaka, Malaysia.

E-mail: ckgan@utem.edu.my

\section{INTRODUCTION}

Mother Earth has started to experience the negative effects of climate change due to the excessive use of fossil energy resources in the past [1]. In this regard, the efficient use of energy resources is one of the crucial aspects that can help to ensure the energy sustainability for our next generations [2-6]. Many countries around the world have established their own energy management guidelines with the primary aim to optimize energy usage and minimize carbon footprints. In Malaysia, the energy audit process is overseen by the Energy Commission (EC) [7]. The Efficient Management of Electrical Energy Regulation 2008 requires the consumers and private licensee who consume or generate more than $3,000,000 \mathrm{kWh}$ of electrical energy for 6 consecutive months to appoint an EC Registered Electrical Energy Manager (REEM) [8]. One of the key roles of REEM is to submit the energy management report to EC that includes the energy auditing and monitoring of the implemented energy saving measures, amongst others in every 6 months. Hence, the use of cost-effective energy monitoring system become essential to track and trace the energy flows particularly for consumer with different sub-meters installed in different plants or buildings.

One of the main challenges for the energy auditing process is the collection of energy consumption data for a specific section of load $[9,10]$. Sophisticated measurement equipment is usually needed to log and store the load consumption data in certain time interval and for certain periods of time. For premises without a functioning SCADA system, this means that the measurement would have to be carried out manually and periodically by the qualified personnel. This will inevitably increase the operational and maintenance costs of a company. In addition, the installation of grid-connected solar PV system on the building or factory rooftops has accelerated in the last few years driven by the strong governmental initiatives $[11,12]$. As a result, the 
monitoring of the solar generation patterns within the building premises has become a necessity. This solar generation information is important as it can be integrated into the existing energy management system to achieve optimize outcome [13]. It is also important to note these data (solar output and load consumption) should be made available in real time.

With the advent of Internet of Things (IoT) technology [14] in the fourth industrial revolution (IR 4.0) era [15], the existing installed digital energy meter that has equipped with industrial communication protocol can be retrofitted to improve its connectivity and observability. This can be achieved by taking the advantage of IoT technology [16]. In light of this, this paper proposes a methodology that embraces the IoT technology so that the existing digital energy meter that has been installed in the buildings can be retrofitted to allow online monitoring. Thus, it provides an alternative from the traditional SCADA online monitoring system which is relatively costly. This remaining part of this paper is organized as follows: Section II discusses the methodology that has been proposed and developed. Actual prototype implementation and field test are presented and discussed in Section III. Finally, conclusions are drawn in Section IV.

\section{RESEARCH METHOD}

The digital power meter nowadays is equipped with the capability of communicating through industrial protocol, such as Modbus, TCP/IP and etc. However different meter manufacturers normally have their own in-house developed proprietary software in order to retrieve and present the meter data in a user-friendly manner. The challenges arise when a building is installed with different types (brands) of meter. Since each brand retrieves the data differently, integrating them together is a significant obstacle. Thus, this project aims to develop a universal wireless-enabled module for digital power meter's Modbus communication protocol. The main driver of this project is to embrace the IoT technology for campus-wide electrical energy monitoring in a cost-effective manner.

Figure 1 shows the overall concept design of UTeM IoT-based electrical energy monitoring system. The hexagonal blocks represent the main buildings inside the campus. The energy consumption of each faculty and centre will be monitored individually. At present, there is no detailed information on the proportion of energy used by the respective faculties in the main campus.

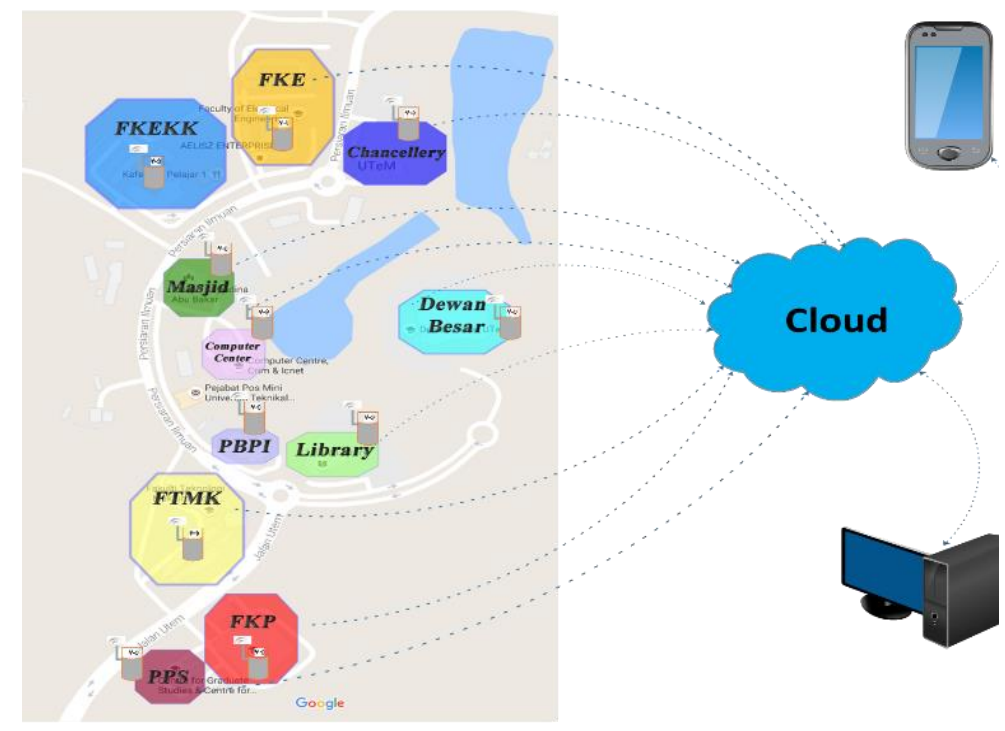

Figure 1. Effects of selecting different switching under dynamic condition

An IoT-based Energy Management System (EMS) that utilizes the industrial Modbus communication protocol has been developed in this project. Figure 2 shows the process flow of how the IoT-based EMS system was developed and implemented. First, the Modbus communication protocol was established to retrieve the data from different digital energy meters installed at different Main Switch Board (MSB). It was then followed by the data decryption that is based on the meter registry code and encryption type with every 10 second time interval. Next, the agent-based cloud controller was developed to log all the retrieved data from all the different meters to the cloud server in "mysql" table format. The logged data is 
then analysed and presented in the customized user interface via the in-house developed web-portal as shown in Figure 3. The energy monitoring portal can be accessed remotely via computer or mobile devices with standard internet connections [17]. In this way, the proprietary format of the specified meter manufacturer can be bypassed as shown in Figure 4.

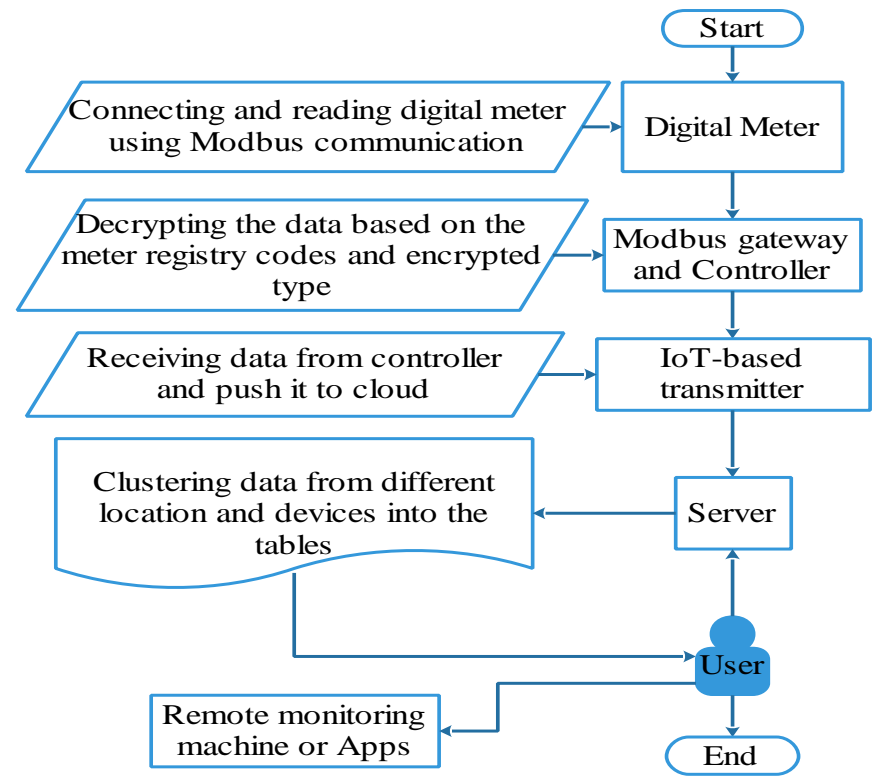

Figure 2. IoT-based Modbus EMS flowchart

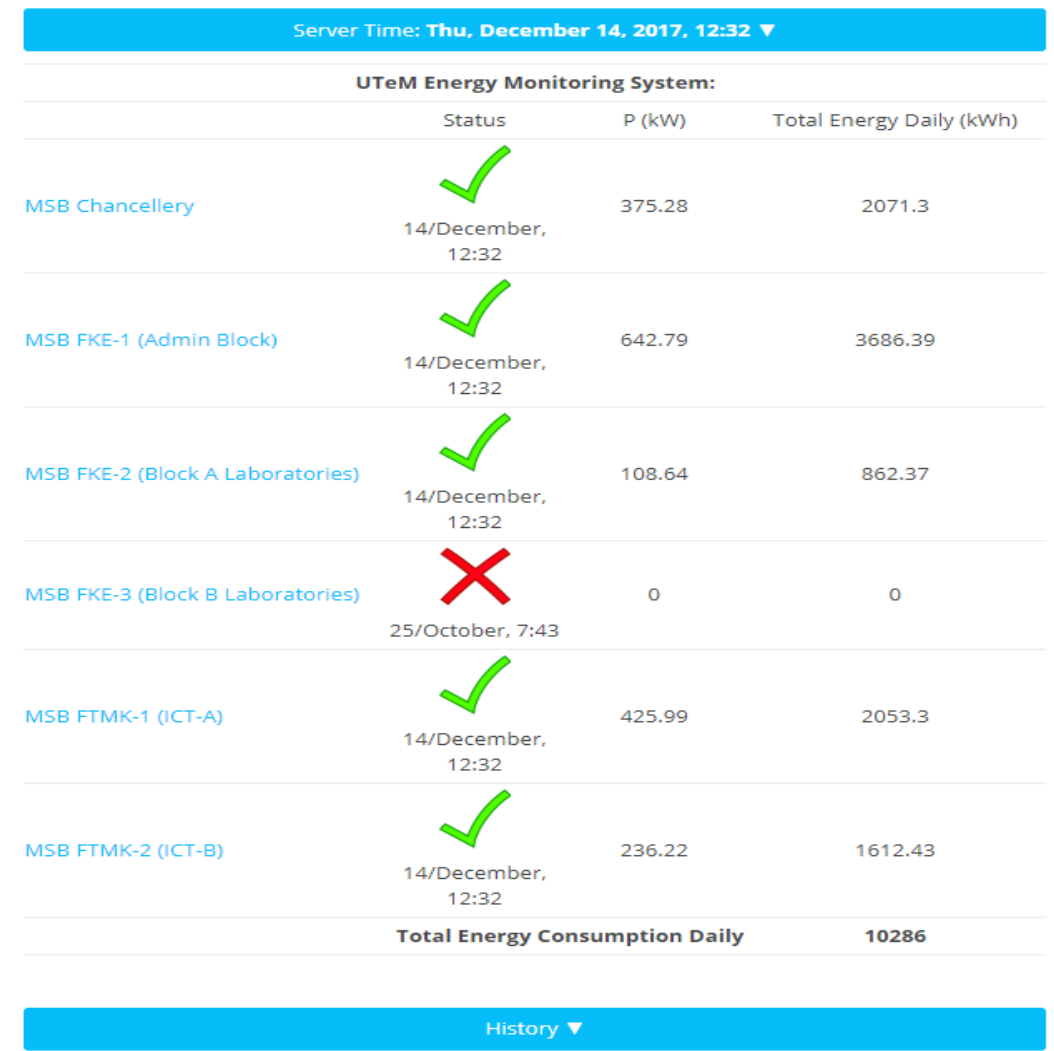




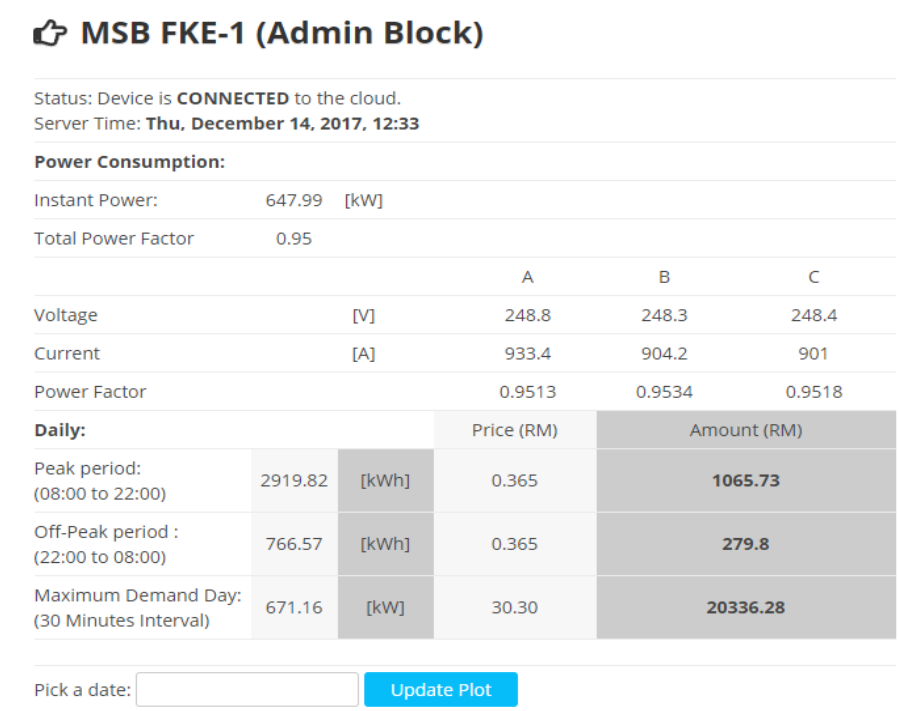

Figure 3. Screen shot of the proposed UTeM energy monitoring web portal

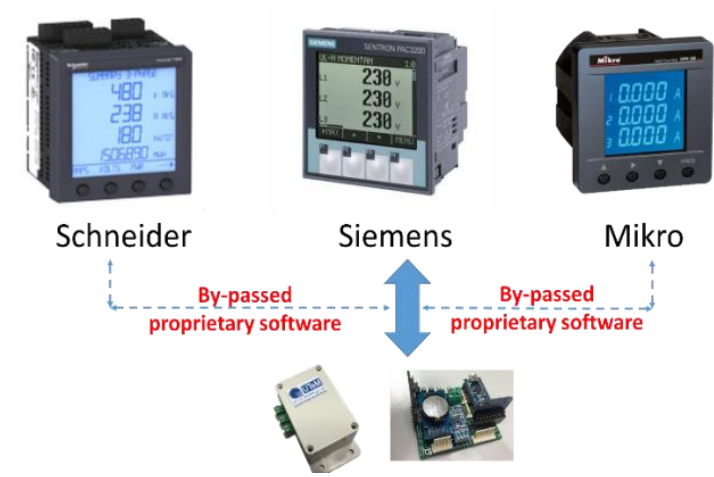

Figure 4. Integrated electrical energy monitoring for various digital meters

\section{RESULTS AND ANALYSIS}

As mentioned in the previous section of the paper, the energy monitoring system that has been developed in this project is able to monitor the power consumption of each faculty buildings. More specifically, the half-hourly load profiles for each of the buildings can be monitored and analysed. This information is valuable to help the university to better understand how the electrical energy was being utilized across the faculties and centres in the main campus. In addition, the behaviour of the user can also be identified from the recorded demand patterns. In this regard, the developed IoT-enabled EMS prototype has been installed at six different MSBs at UTeM main campus. The lists are as follows:
a. MSB Chancellery
b. MSB FKE-1 (Administration block)
c. MSB FKE-2 (Block A laboratories)
d. MSB FKE-3 (Block B laboratories)
e. MSB FTMK-1 (ICT A)
f. MSB FTMK-2 (ICT B)

As shown in Figure 3, the monitored data include the voltage and current of each phase, together with the associated power factor. Furthermore, the energy usage is also calculated in the monetary form based on the UTeM's TNB tariff category. Both the energy (kWh) and maximum demand (kW) charges are included. More importantly, all the data are stored in the virtual cloud server and can be retrieved easily when needed.

Figure 5 shows the main campus's Chancellery building power consumption data captured by the IoT-enabled EMS system on 3 December 2017. This load profile shows the baseload of the building during 
the weekend where chillers were switched OFF. The average half-hourly baseload power consumption is approximately $49 \mathrm{~kW}$ during weekend. During working weekdays when the chillers are switched ON, the load profiles pattern are as shown in Figure 6. It can be seen from the figure that the building demand starts to pick up at 7:30am and stabilizes at 9.30am. This is the period for the chiller system to achieve the required temperature set-point in the building. The building load decreases drastically at $5.30 \mathrm{pm}$ following the shutting down of the chiller system.

Further analysis was carried out to compare the energy saving of the same building before and after the energy saving measure has been taken, i.e. by running only one chiller. The saving is significant as can be seen in Figure 6 of which the peak demand of the building decreases from approximately $600 \mathrm{~kW}$ to $380 \mathrm{~kW}$. The daily energy saving is $2,441.5 \mathrm{kWh}$ which represents $21 \%$ of energy saving for the implemented energy saving measure. Such a detail of the building load profiling before and after the energy saving measure would not been possible before the installation of the proposed IoT EMS prototype. Otherwise, dedicated equipment will need to be installed by the qualified personnel in the MSB room for certain period of time, which is costly and not efficient. Therefore, the development of such IoT EMS system would help the university to identify opportunities for energy saving in the buildings.

Figure 7 shows the energy consumption pattern for FTMK building during weekend and working day. Similarly to the Chancellery building, the peak demand during weekday was contributed by the chillers. The recoded peak demand is slightly higher than $300 \mathrm{~kW}$ as recorded at 11:30am. It is interesting to observe that there was an obvious drop in peak demand during the afternoon lunch hour (12:30-2:00pm). This decrease in demand might be contributed by the good behaviour of the users in switching off the electrical load when they are not in use. Even though similar decrease in demand during the lunch hour can be seen in Chancellery building, the order of magnitude is relatively small as compared to FTMK building. Thus, the user behaviour is one of the important factors that an organization should focus to reduce the total energy consumption in a more holistic way.

The administration block of the Faculty of Electrical Engineering (FKE) has relatively high energy consumption as compared to the other buildings. The Saturday (2/12/2017) and Tuesday (5/12/2017) load profile of FKE administration block is as shown in Figure 8. The demand remains above $600 \mathrm{~kW}$ during the peak hour period.

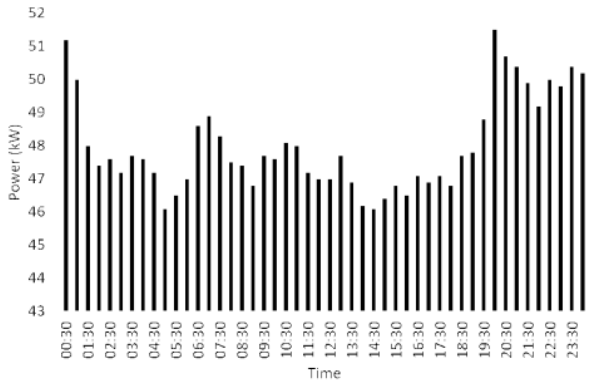

Figure 5. Active power consumption chancellery 3/12/2017 (weekend) (2304.5 kWh)

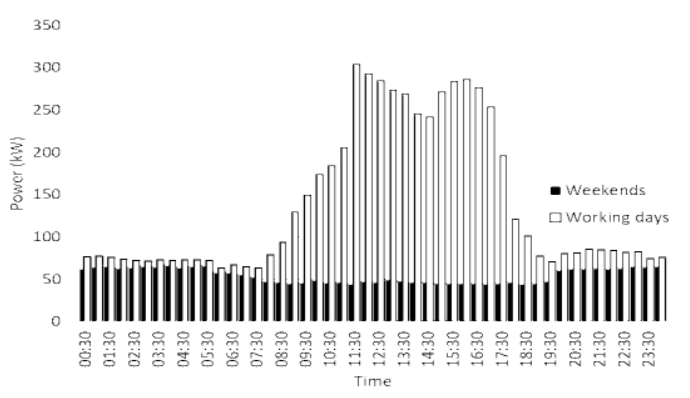

Figure 7. Active power consumption FTMK (ICT\#B) 2/12/2017 (weekend) (2600 kWh) and $5 / 12 / 2017$ (weekday) (6664.2 kWh)

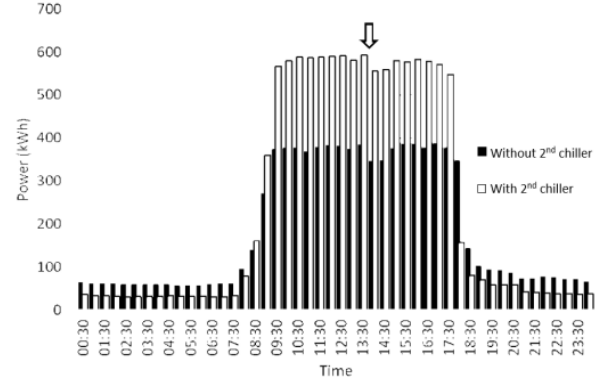

Figure 6. Active power consumption chancellery 13/06/2017 (weekday) before (11636.4 kWh) after shutting off chiller $(5 / 12 / 2017) \# 2(9194.9 \mathrm{kWh})$

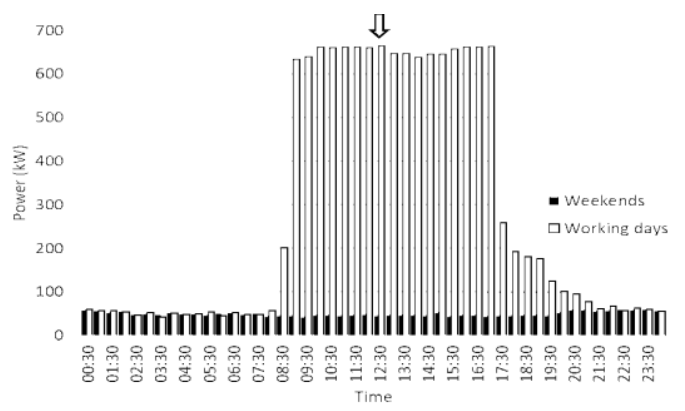

Figure 8. Active power consumption FKE (Admin Block) 2/12/2017 (weekend) (2398.9 kWh) and $5 / 12 / 2017$ (weekday) (13712.5 kWh) 
Figure 9 shows the power consumption profiles recorded from the "MSB FKE-2 (Block A laboratories)" that supplies the laboratory electrical energy usage during weekend. It can be clearly seen from Figure 9 that the total power drawn from this MSB starts to decrease gradually at 7.30 am and reaches its minimum at around 12.30 to $1.00 \mathrm{pm}$. This $\mathrm{U}$ shape load profile is as expected as one of the laboratories it supplies has installed with $24 \mathrm{~kW}$ grid-connected solar PV system [2]. During sunny day, the solar system will have the highest electricity generation at solar noon $(1.00 \mathrm{pm}$ Melaka time). The solar irradiance profile of the same day (2/12/2017) is also shown in the Figure 9. This explains the trough of the load profile. Without the solar PV system, the load profile would have been rather flattening, similar to the one as shown in Figure 8.

From this observation, it is important to note that the solar energy generated from the $24 \mathrm{~kW} \mathrm{PV}$ system is entirely self-consumed within the building even during the baseload condition (weekend). In other words, there is no excess solar energy produced that would have been otherwise exported to the other nearby buildings. The load profiles for this MSB supplying Block A laboratories during weekday is as shown in Figure 10. This profile is the net energy consumption after taking into consideration the solar lab PV generation.

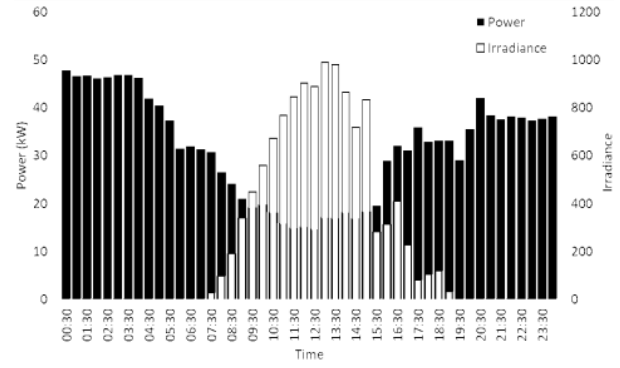

Figure 9. Active power consumption FKE (block a laboratories) (weekend) (1517.8 kWh) and solar irradiance at UTeM on 2/12/2017

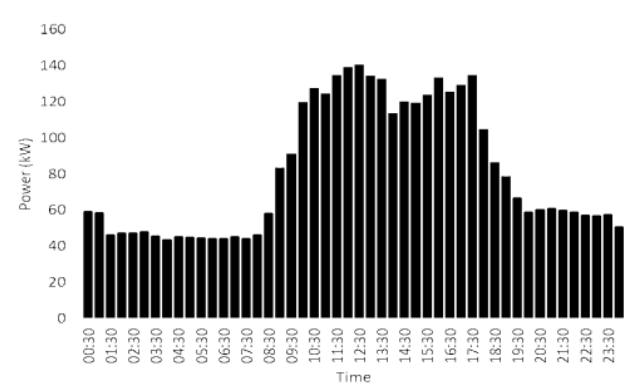

Figure 10. Active power consumption FKE (block a laboratories) 5/12/2017 (weekday) (3920.2 kWh)

\section{CONCLUSION}

This paper presents an UTeM in-house developed energy monitoring solution which can monitor the campus-wide electricity cost-effectively by embracing the Internet of Things technology. Actual field data which were captured at three different MSB locations at UTeM main campus have been presented and analysed. This IoT-enabled solution allows the monitoring of electricity usage at the sub-metering levels. In addition, the time series load profiles that were captured on half-hourly basis can provide useful information for the energy manager to implement the relevant energy saving measures. The quantification of the electricity bill saving before and after the implementation of certain energy saving measure can also be carried out easily.

\section{ACKNOWLEDGEMENTS}

The authors would like to gratefully acknowledge the funding provided by the Universiti Teknikal Malaysia Melaka under JURNAL/2018/FKE/Q00012.

\section{REFERENCES}

[1] T. Partridge et al., "Seeing futures now: Emergent US and UK views on shale development, climate change and energy systems," Glob. Environ. Chang., vol. 42, pp. 1-12, 2017.

[2] P. H. Tan, C. K. Gan, and K. A. Baharin, "Techno-economic analysis of rooftop PV system in UTeM Malaysia," in 3rd IET International Conference on Clean Energy and Technology (CEAT), pp. 1-6. 2014.

[3] M. Shamshiri, C. K. Gan, and R. Omar, "Assessment of distribution networks performance considering residential photovoltaic systems with demand response applications," J. Renew. Sustain. Energy, vol. 9, no. 4, 2017.

[4] "Assessment of Achievable Potential from Energy Efficiency and Demand Response Programs in the U.S. (2010-2030), Electric Power Research Institute (EPRI) [Online] : http:/www.epri.com/abstracts/pages/productabstract.aspx?ProductID=000000000001016987.”.

[5] A. H. Hashim, A. K. Khairuddin and J. B. Ibrahim, "Integration of renewable energy into grid system-the Sabah Green Grid," 2015 IEEE Eindhoven PowerTech, Eindhoven, 2015, pp. 1-6. 
[6] SEDA, "Sustainable Energy Development Authority of Malaysia (SEDA) Annual Report 2015," 2015.

[7] "Electrical Energy Audit Guidelines," Suruhanjaya Tenaga (Energy Commission), 2016. [Online]. Available: http://www.st.gov.my/index.php/en/all-publications/item/719-electrical-energy-audit-guidelines-for-building.

[8] Suruhanjaya Tenaga Malaysia, "Efficient Management of Electrical Energy Regulation 2008 [P.U. (A)444]," pp. $1-28,2008$.

[9] P. Thollander and J. Palm, "Improving energy efficiency in industrial energy systems: An interdisciplinary perspective on barriers, energy audits, energy management, policies, and programs". Springer Science \& Business Media, 2012.

[10] Y. Agarwal and T. Weng, "Understanding the Role of Buildings in a Smart Microgrid."

[11] B. A. Malek, "Renewable energy development in Malaysia," 34th APEC Expert Gr. new Renew. energy Technol. (EGNRET), Kuala Lumpur, pp. 26-27, 2010.

[12] A. Ipakchi and F. Albuyeh, "Grid of the future," in IEEE Power and Energy Magazine, vol. 7, no. 2, pp. 52-62, March-April 2009.

[13] H. Outhred and E. Systems, "Meeting the Challenges of Integrating Renewable Energy into Competitive Electricity Industries," Renewable Energy Integration Law Project ,2007.

[14] J. Mona, "Data Communication in Internet of Things: Vision, Challenges and Future Direction," Telkomnika (Telecommunication Computing Electronics and Control), vol. 16, no. 5, pp. 2057-2062, 2018.

[15] "Industry 4.0." [Online]. Available: http://www.miti.gov.my/index.php/pages/view/industry4.0?mid=559.

[16] Y. Abdul, A. S. Aldeen, and K. N. Qureshi, "New Trends in Internet of Things, Applications, Challenges, and Solutions," Telkomnika (Telecommunication Computing Electronics and Control), vol. 16, no. 3, pp. 1693-6930, 2018.

[17] M. Shamshiri, C. K. Gan, J. Safaei, R. B. Omar, M. R. B. A. Ghani, and M. R. Tamjis, "Smart grid application via home automation system," Int. J. Appl. Eng. Res., vol. 10, no. 24, pp. 43930-43933, 2015. 